can be seen on echocardiogram. A positive troponin is a common finding with a subsequent normal coronary angiogram. Incidental paraproteins are prevalent in up to $8 \%$ of this population and it is important to obtain a tissue diagnosis to rule out AL amyloidosis. With supportive management medium term outcomes are good.

\section{PROGNOSTIC UTILITY OF CALCULATED PLASMA VOLUME STATUS IN CHRONIC HEART FAILURE}

doi:10.1136/heartjnl-2011-300198.104

${ }^{1} \mathrm{H}$ Z Ling, ${ }^{1} \mathrm{~N}$ Aung, ${ }^{1,2} \mathrm{~J}$ Flint, ${ }^{1,2} \mathrm{~S}$ Aggarwal, ${ }^{1,2} \mathrm{~S}$ Weissert, ${ }^{1,2} \mathrm{~A}$ Cheng, ${ }^{3} \mathrm{D}$ P Francis, ${ }^{3} \mathrm{~J}$ Mayet, ${ }^{1,2} \mathrm{M}$ Thomas, ${ }^{1,2} \mathrm{~S}$ Woldman, ${ }^{1,2,4} \mathrm{D} 0$ Okonko. ${ }^{1}$ University College London Hospital, London, UK; ${ }^{2}$ The Heart Hospital, London, UK; ${ }^{3}$ International Center for Circulatory Health, NHLI, Imperial College London, London, UK; ${ }^{4} \mathrm{NHLI}$ Imperial College London, London, UK

Background Plasma volume (PV) expansion is a hallmark feature of worsening heart failure that is notoriously underestimated by clinical examination. While radioisotope assays optimally quantify PV status, numerous haemodialysis-based equations also exist for its estimation. The prognostic utility of such formulas in chronic heart failure (CHF) is unknown.

Methods We analysed the relation between estimated PV status and mortality in 246 outpatients with CHF (mean $( \pm S D)$ age $67 \pm 13$ years, NYHA class $2 \pm 1$, LVEF $28 \pm 8 \%$ ). PV status was calculated (Hakim RM, et al) by subtracting the patients actual PV ((1-haematocrit) $\times$ $(a+(b \times$ weight $)) ; a$ and $b$ are gender-specific constants $)$ from their ideal PV ((c $\times$ weight); $c=$ gender-specific constant).

Results Median ( \pm IOR) PV status was-261 $\pm 550 \mathrm{ml}$ with $78 \%$ and $21 \%$ of patients having PV contraction and expansion, respectively. Patients with PV excess had significantly higher creatinine and lower albumin levels. Over a median follow-up of $13 \pm 16$ months, $36(15 \%)$ patients died. PV status predicted mortality (HR 1.001, 95\% CI 1.001 to $1.002, p=0.001$ ) in a graded fashion (Abstract 104 figure $1 \mathrm{~A}$ ) and did so independently of NYHA class, LVEF, weight, haematocrit and creatinine. A PV status $\leq-178 \mathrm{ml}$ optimally predicted survival (ROC AUC $0.68, p=0.0007$ ) and conferred a $75 \%$ reduced hazard for death (HR 0.16, 95\% CI 0.07 to 0.37, p<0.0001, Abstract 104 figure 1B).
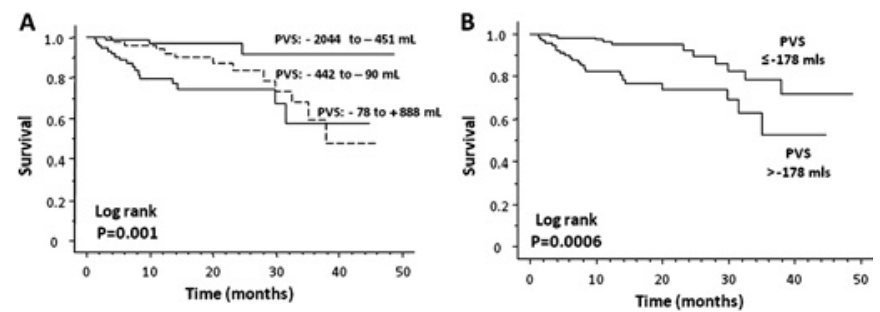

Abstract 104 Figure 1

Conclusions Calculating plasma volume status in CHF patients appears prognostically useful and suggests that dehydration is better tolerated than volume excess in these individuals and that targeting therapy to achieve a plasma volume status $\leq 178 \mathrm{ml}$ might increment survival.

\section{CLINICAL AND ECHOCARDIOGRAPHIC DETERMINANTS OF N-TERMINAL PRO B-TYPE NATRIURETIC PEPTIDE LEVEL IN PATIENTS WITH STABLE CHRONIC OBSTRUCTIVE AIRWAYS DISEASE: A PROSPECTIVE OBSERVATIONAL STUDY OF 140 PATIENTS}

doi:10.1136/heartjnl-2011-300198.105

${ }^{1} \mathrm{C}$ P Gale, ${ }^{2} \mathrm{~J}$ White, ${ }^{2} \mathrm{~A}$ Hunter, ${ }^{3} \mathrm{~J}$ Owen, ${ }^{4} \mathrm{~J}$ Watson, ${ }^{5} \mathrm{R}$ Pearson, ${ }^{4} \mathrm{I}$ Holbrook, ${ }^{6} \mathrm{~N}$ Durham, ${ }^{6} \mathrm{M}$ Pye. ${ }^{1}$ Division of Biostatistics, University of Leeds, Leeds, UK;
${ }^{2}$ Department of Respiratory Medicine, York Hospitals NHS Foundation Trust, York, UK; ${ }^{3}$ Department of Echocardiography, York Hospitals NHS Foundation Trust, York, UK; ${ }^{4}$ Department of Biochemistry, York Hospitals NHS Foundation Trust, York, UK; ${ }^{5}$ Leeds Teaching Hospitals, Leeds, UK; ${ }^{6}$ Department of Cardiology, York Hospitals NHS Foundation Trust, York, UK

Background Brain natriuretic peptides have been shown to be reliable indicators of left ventricular failure and markers of risk in cardiac disease. However, patients with chronic obstructive pulmonary disease (COPD) are also known to have elevated concentrations of brain natriuretic peptides in the absence of overt cardiac disease, likely due to right ventricular strain. This has been shown to have prognostic value and has a potential role in the management of the condition; for example, it has been suggested that it could be used to guide the initiation of non-invasive ventilation. The aim of this study was to identify clinical and echocardiographic determinants of the polypeptide N-terminal proBrain Natriuretic Peptide (NT pro-BNP) in patients with stable COPD.

Method Arterial blood gases, plasma NT pro-BNP and transthoracic echocardiographic parameters were studied in 140 patients with stable COPD attending a respiratory outpatient clinic.

Results Of the 140 patients, 65 (46\%) were male, $26(19 \%)$ received home oxygen therapy, 115 (82\%) were current smokers, 38 (27\%) were prescribed diuretics and $15(11 \%)$ had a left ventricular ejection fraction $<45 \%$. Patients with cor pulmonale $(n=6)$ were more likely to have left ventricular systolic dysfunction $(p<0.001)$, reduced tricuspid annular plane systolic excursion $(p=0.017)$ and higher pulmonary artery systolic pressures $(p=0.01)$. The median (IOR) NT pro-BNP concentration was 16.2 (25.4) pmol/l. Concentrations were significantly higher in those with a dilated left atrium, aortic stenosis, left ventricular systolic dysfunction, right ventricular impairment, atrial fibrillation and those prescribed diuretics and ACE inhibitors. Significant predictors of NT pro-BNP were a dilated left atrium, aortic stenosis and left ventricular systolic dysfunction. NT Pro-BNP was an excellent discriminator of RV impairment (C statistic $=0.90$ )

Conclusions NT pro-BNP readily identifies patients with stable COPD who have right ventricular dysfunction. However, several other clinical variables also associated with increased NT pro-BNP concentrations are prevalent in this population. This is likely to confound clinical decision making.

\section{CHF PATIENTS ARE VITAMIN D DEFICIENT AND HYPERPARATHYROID, WITH LEVELS OF EACH RELATED TO MARKERS OF SEVERITY}

doi:10.1136/heartjnl-2011-300198.106

${ }^{1} \mathrm{G}$ A Begg, ${ }^{1} \mathrm{~L}$ Kearney, ${ }^{2} \mathrm{~A}$ C Wheatcroft, ${ }^{1} \mathrm{R}$ Byrom, ${ }^{1} \mathrm{~S}$ Barnes, ${ }^{1} \mathrm{~J}$ Gierula, ${ }^{2} \mathrm{~J}$ Barth, ${ }^{2} \mathrm{R}$ Cubbon, ${ }^{2} \mathrm{M}$ T Kearney, ${ }^{2} \mathrm{~K}$ K Witte. ${ }^{1}$ Leeds General Infirmary, Leeds, UK; ${ }^{2}$ University of Leeds, Leeds, UK

Background The vitamin D-parathyroid (PTH) axis is increasingly recognised as potentially being involved with many of the features of the syndrome of CHF. We wanted to explore the relationship between vitamin D and PTH levels in a group of CHF patients and relate these to markers of severity.

Methods We analysed serum 25(OH) vitamin D3 levels in 406 consecutive attendees of the Leeds Advanced Heart Failure clinic (310 men) and correlated these to clinical markers of severity.

Results Mean age (SE) was 69 (3) years, mean left ventricular ejection fraction (LVEF) 31 (2)\%, mean serum creatinine $117 \mu \mathrm{mol} / 1$ (2.4), median vitamin D levels (IOR) $30(20-43) \mathrm{nmol} / \mathrm{l}$ (normal for skeletal health $>75 \mathrm{nmol} / \mathrm{l}$ ) and median parathyroid levels 8.8 $(6.2-13.5) \mathrm{pmol} / \mathrm{l}$ (normal $<6.5 \mathrm{pmol} / \mathrm{l}$ ). Aetiology was ischaemic 
heart disease in $63 \%$ and $23 \%$ had diabetes mellitus. Patients were optimally treated (84\% on $\beta$-blockers, $88 \%$ on ACE inhibitors, and $46 \%$ on spironolactone). The mean daily dose of furosemide was 60 (3) mg. Very few patients (5\%) were sufficient in vitamin D. Patients with worse symptoms as measured by NYHA status had lower vitamin D levels and higher PTH levels (Abstract 106 figures 1 and 2 ). There was also a negative relationship between furosemide dose and vitamin D (Abstract 106 figure 3) and, in an unselected subset of 160 patients (mean peak oxygen uptake $\left(\mathrm{pVo}_{2}\right) 16.6(0.5) \mathrm{ml} / \mathrm{kg}$ / $\mathrm{min}$ ), there was a positive relationship between $\mathrm{pVo}_{2}$ and vitamin $\mathrm{D}$ (Abstract 106 figure 4). Patients with diabetes had lower vitamin D levels than non-diabetics $(p<0.001)$ and there was a negative correlation between vitamin $\mathrm{D}$ and fasting glucose levels $(\mathrm{r}=0.13$; $\mathrm{p}=0.02$ ). There was no relationship between vitamin $\mathrm{D}$ levels and age, calcium, creatinine or CRP, and no differences between those patients taking and those not taking $\beta$-blockers and ACE inhibitors. In 8 unselected patients we found a negative relationship between tumour necrosis factor-alpha (TNF- $\alpha$ ) levels and vitamin $\mathrm{D}(\mathrm{r}=0.62 ; \mathrm{p}=0.05)$. Although there was no relationship between vitamin D levels and baseline LVEF, in a subgroup of 150 patients followed up one year after titration to optimal CHF therapy, there was a significant positive relationship between change in LV dimensions and vitamin $\mathrm{D}$ levels at the time of the baseline scan $(p<0.05)$

Conclusions The vitamin D-PTH axis is abnormal in CHF, related to the severity of the condition. Our data suggest that reverse remodelling in response to optimal drug titration is greater in those with higher vitamin $\mathrm{D}$ levels. Whether vitamin $\mathrm{D}$ deficiency is causally related to CHF remains unknown and requires a long-term, randomised, placebo-controlled study in CHF patients with efficacy and mechanistic outcomes, using a dose of vitamin $\mathrm{D}$ capable of normalising both vitamin D and PTH levels.

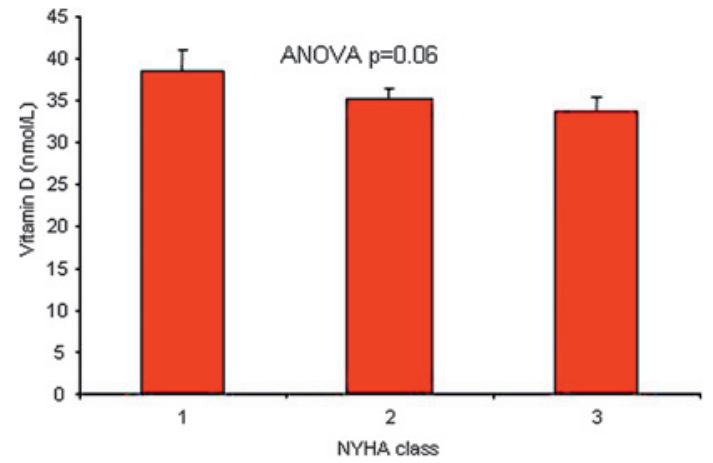

Abstract 106 Figure 1

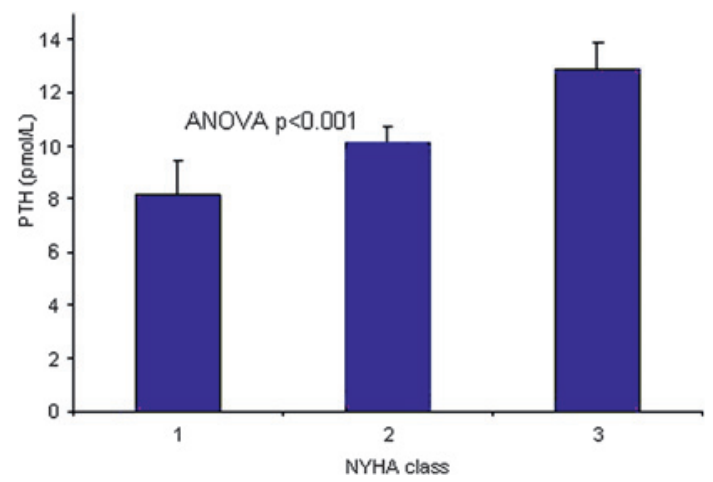

Abstract 106 Figure 2

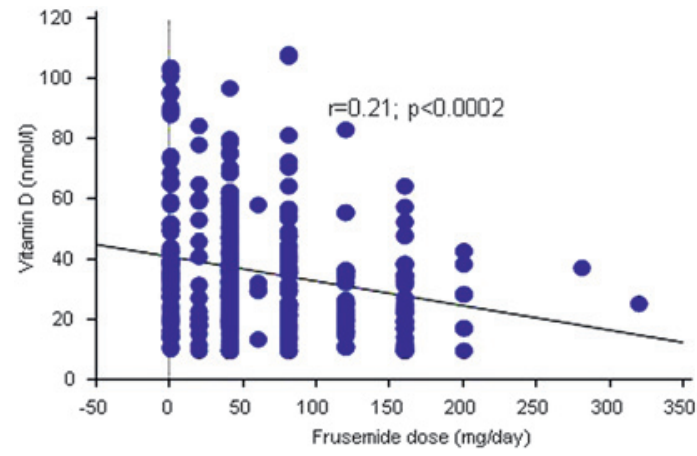

Abstract 106 Figure 3

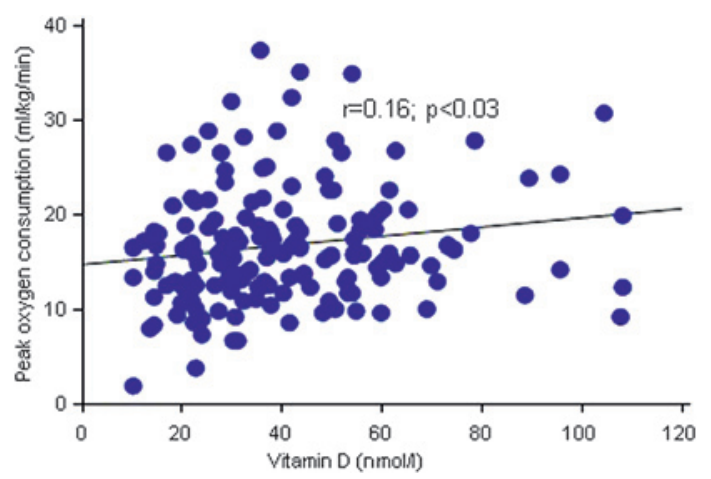

Abstract 106 Figure 4

\section{EXPANSION OF THE RED CELL DISTRIBUTION WIDTH AND EVOLVING IRON DEFICIENCY AS PREDICTORS OF POOR OUTCOME IN CHRONIC HEART FAILURE}

doi:10.1136/heartjnl-2011-300198.107

${ }^{1} \mathrm{~N}$ Aung, ${ }^{1} \mathrm{H} \mathrm{Z}$ Ling, ${ }^{1,2} \mathrm{~S}$ Aggarwal, ${ }^{1,2} \mathrm{~J}$ Flint, ${ }^{1,2} \mathrm{~S}$ Weissert, ${ }^{1,2} \mathrm{~A}$ Cheng, ${ }^{1} \mathrm{~T}$ Richards, ${ }^{3} \mathrm{D}$ P Francis, ${ }^{3} \mathrm{~J}$ Mayet, ${ }^{1,2} \mathrm{M}$ Thomas, ${ }^{1,2,4} \mathrm{D} 0$ Okonko. ${ }^{1}$ University College London Hospital, London, UK; ${ }^{2}$ The Heart Hospital, London, UK; ${ }^{3}$ International Center for Circulatory Health, NHLI Imperial College London, London, UK; ${ }^{4}$ NHLI Imperial College, London, UK

Background Red cell distribution width (RDW) is a surrogate of many aberrations (inflammation, malnutrition, iron deficiency (ID)) that may drive chronic heart failure (CHF) progression. While an elevated RDW and iron deficiency at baseline predict mortality in $\mathrm{CHF}$, little is known about the prognostic implications of their temporal trends.

Methods We analysed the relation of red cell indices on first consultation and over time with mortality in 274 outpatients with CHF (mean $( \pm S D)$ age $70 \pm 14$ years, LVEF $28 \pm 8 \%$, NYHA class $2 \pm 1,54 \%$ ischaemic). The combination of a rising RDW and a falling mean cell volume (MCV) identified evolving ID.

Results On initial consultation, an RDW $>15 \%, \mathrm{Hb}<12.5 \mathrm{~g} / \mathrm{dl}$, and MCV $<80 \mathrm{fl}$ were evident in $41 \%, 46 \%$, and $8 \%$ of patients. Over a median ( \pm IOR) follow-up of $15 \pm 17$ months, 60 (22\%) patients died. On Cox proportional hazards analyses, a higher RDW independently predicted increased mortality (HR 1.21, $\mathrm{p}<0.0001)$. Over time, $51 \%, 58 \%, 40 \%$, and $23 \%$ of patients had a rise in RDW, a fall in $\mathrm{Hb}$, a fall in $\mathrm{MCV}$, and evolving ID, respectively. A rising RDW predicted death (HR 1.18, $\mathrm{p}=0.002$ ) independently of baseline RDWs and changes in $\mathrm{Hb}$, with an absolute increase $>1 \%$ conferring a twofold escalated risk of mortality (Abstract 107 figure 1A). Evolving ID was also associated with poorer survival (HR 2.89, $\mathrm{p}<0.0001$, Abstract 107 figure 1B). 